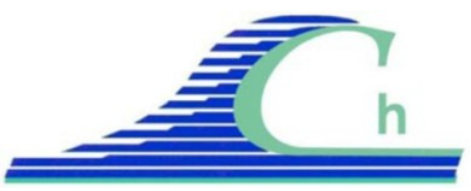

XII ${ }^{\text {ìmes }}$ Journées Nationales Génie Côtier - Génie Civil

Cherbourg, 12-14 juin 2012

DOI:10.5150/jngcgc.2012.118-M C Editions Paralia CFL

disponible en ligne - http://www.paralia.fr - available online

\title{
Etude de la durabilité des sédiments de dragage du port d'Honfleur traités au ciment et additifs
}

\author{
Salim MEZAZIGH ${ }^{1}$, Ernesto SILITONGA ${ }^{1}$, Xue LEI $^{1}$, Xiaohui BAI ${ }^{1}$
}

1. Université de CAEN, Laboratoire "Morphodynamique Continentale et Côtière" M2C 24, rue des Tilleuls 14000, Caen, France.

salim.mezazigh@unicaen.fr

\section{Résumé :}

Les opérations de dragage sont nécessaires pour maintenir la navigation dans les cours d'eau et l'accès aux ports. Chaque année, 100 millions de tonnes de matériaux sont dragués dans le monde entier. Ces matériaux peuvent contenir une quantité variable de matière organique et différents polluants.

En raison de l'approche de développement durable adoptée par plusieurs pays, la valorisation des sédiments de dragage a acquis une acceptation dans différents domaines comme le génie civil, l’agriculture.

Des industriels mettent en avant le besoin d'étendre l'utilisation de la technique de solidification/stabilisation aux sédiments inorganiques contenant un niveau plus ou moins important de polluants organiques. La dispersion des polluants dans l'environnement des sédiments stabilisés par des liants hydrauliques, dépendra de la solubilité des espèces polluantes et aussi des qualités intrinsèques du matériau solidifié/stabilisé.

Dans ce contexte, l'article présente l'étude réalisée pour l'élaboration d'un matériau de construction basé sur des sédiments dragués ainsi que l'évaluation de l'influence des liants sur le comportement mécanique et la durabilité des sédiments traités.

Mots-clés : Sédiments marins - Valorisation - Durabilité - Fumée de silice - Matériau routier - Compression simple - Mouillage-séchage - Gel-Dégel - Lixiviation

\section{Abstract:}

The dredging is needed to maintain navigation in the waterways and port access. Each year 100 million tons of material are dredged in the world. These materials may contain variable amounts of organic matter and other pollutants.

Due to the sustainable development approach adopted by several countries, valorization of dredged sediment has gained acceptance in various fields such as civil engineering, agriculture.

Manufacturers highlight the need to expand the use of the technique of solidification/stabilization with inorganic sediments containing a more or less of organic pollutants. The dispersion of pollutants in the environment of sediments stabilized by hydraulic binders, depend on the solubility of the pollutant species and also the intrinsic qualities of the material solidified/stabilized. 
In this context, the paper presents the study for the development of a construction material based on dredged sediments and the evaluation of the influence of binders on the mechanical behavior and durability of the treated sediment.

Keywords: Marine sediments - Valorization - Sustainability - Silica fume - Road material - Simple compression - Wetting-drying - Freezing and thawing - Leaching

\section{Introduction}

En France, l'activité de dragage représente environ 50 millions de $\mathrm{m}^{3}$ de matériaux chaque année, avec une forte contribution des grands complexes portuaires dont le maintien et le développement de l'activité sont fortement dépendants.

La filière immersion représente en France plus de 99\% des matériaux dragués chaque année, soit entre 20 à 40 millions de $\mathrm{m}^{3}$ de matériaux. Dès que cette filière est exclue, les matériaux doivent être destinés à terre, et entrent de ce fait dans la réglementation déchets. Mais le choix de la filière n’est pas toujours aisé en raison du contexte réglementaire et des incertitudes sur les impacts environnementaux.

Cette étude vise donc la production d'une solution de valorisation durable des sédiments de dragage portuaires contaminés de type vaseux.

\section{Caractéristiques physico-chimiques des sédiments}

Les sédiments de la présente étude sont issus d’un dragage réalisé dans le port d'Honfleur. Une des premières étapes de la caractérisation d'un sol est d'évaluer sa courbe granulométrique. Les mesures ont été effectuées sur une granulométrie laser. Le tableau 1 résume la nature granulométrique des sédiments du port d’Honfleur. Il apparaît que le sédiment étudié est essentiellement limoneux. De plus, d’après les résultats présentés dans le tableau 1 , on constate que la teneur en matière organique des sédiments tourne autour d'une moyenne de $4,24 \%$ ce qui permet de classer les sédiments en sédiments organiques ( $\mathrm{MO}>3)$.

La teneur en eau joue un rôle très important par la suite dans le traitement des sédiments. Dans le cas de notre sédiment, plusieurs mesures de teneur en eau ont été effectuées. Le tableau en donne une valeur moyenne. On observe que la teneur en eau naturelle après dragage des sédiments est élevée. Elle est en moyenne de 120\% (figure 1, tableau 1). Dans notre cas, selon la classification du GTR (1992), les sédiments étudiés appartiennent à la classe $\mathrm{A}_{1}$ qui désigne les limons peu plastiques. La valeur au bleu de méthylène permet de préciser cette classification AFNOR NF P 94-068, (1998). Par ailleurs, le diagramme de Casagrande (figure 2), classe le sédiment en limon peu plastique à très plastique.

Trois essais au bleu de méthylène ont été réalisés sur des échantillons. La valeur moyenne des résultats obtenus est présentée dans le tableau 1. Ces résultats confirment la classification des sédiments utilisés en limons sensibles à l'eau. 


\section{XII ${ }^{\text {èmes }}$ Journées Nationales Génie Côtier - Génie Civil \\ Cherbourg, 12-14 juin 2012}

Tableau 1. Caractéristiques physico-chimiques des sédiments du port d'Honfleur.

\begin{tabular}{ll}
\hline Caractérisation & (\%) \\
\hline Nature granulométrique & \\
\hline Fraction sableuse $(>63 \mu \mathrm{m})$ & 13 \\
Fraction silteuse $(2$ à $63 \mu \mathrm{m})$ & 76 \\
Fraction argileuse $(<2 \mu \mathrm{m})$ & 11 \\
Limites d'Atterberg & \\
Limite de liquidité, $W L$ & 46,6 \\
Limite de plasticité, $W P$ & 35,3 \\
Indice de plasticité, IP & 11,3 \\
& \\
Teneur en matière organique, MO & 4.24 \\
Teneur en eau initiale, Wi & 120 \\
Valeur moyenne au bleu de méthylène VBS & 0,21 \\
\hline
\end{tabular}

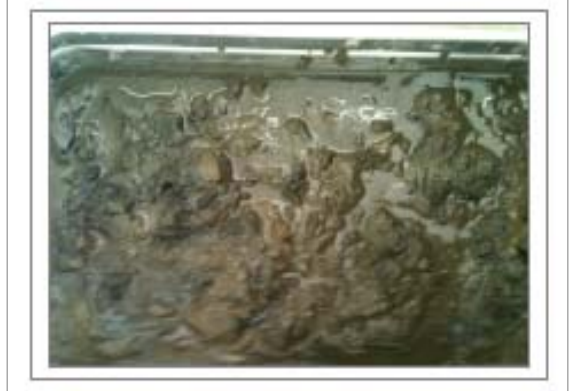

Figure 1. Echantillon des sédiments dragués au port d'Honfleur.

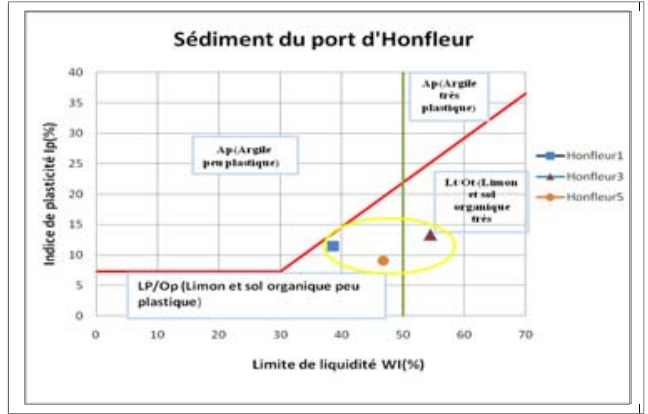

Figure 2. Diagramme de plasticité et de classification des sols fins.

\section{Procédé de confection des éprouvettes}

Les échantillons étudiés ont été réalisés dans des moules cylindriques de diamètre 40 $\mathrm{mm}$ et de hauteur $80 \mathrm{~mm}$. L'élancement est de 2 (figure 3). Pour faciliter le démoulage, les moules sont fendus sur une génératrice et maintenus fermés à l'aide de colliers métalliques. Les mottes de sédiments obtenues après séchage sont concassées afin d'obtenir un maximum fin. Un tamisage à $2 \mathrm{~mm}$ est nécessaire par la suite avant de confectionner les éprouvettes.

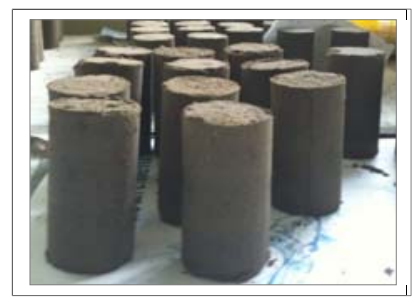

Figure 3. Les échantillons $4 \times 8 \mathrm{~cm}$. 
Les échantillons sont confectionnés dans les moules en trois couches. Chaque couche est compactée par 6 coups de dame afin de simuler un compactage de Proctor Normal. Le mode opératoire s'inspire de celui des normes relatives à la confection d'éprouvettes de mortier normalisé. Chaque constituant est pesé avec une précision de +/-0.5 g et est malaxé à vitesse lente.

Les éprouvettes sont démoulées après la préparation. Elles sont conservées à la température ambiante du laboratoire. Toutefois, aucune anomalie de gonflement ou de fissuration n’a été observée.

Les différentes formulations étudiées sont les suivantes (LEI, 2011 ; BAI, 2010):

- Formulation F1: 5\% sable; 3\% chaux; 3\% ciment; 0,5\% fumée de silice,

- Formulation F2: 5\% sable; 3\% chaux; 5\% ciment; 0,5\% fumée de silice.

\section{Essais physico-mécaniques}

\subsection{Essais en compression simple}

L'essai de résistance en compression simple a été réalisé pour étudier l'effet des liants utilisés sur les paramètres de résistance mécanique des sédiments traités.

Les résultats des deux groupes d'essais (ciment, chaux vive et fumée de silice) sont présentés dans le tableau 2. La figure 4 présente l'évolution avec le temps de la résistance en compression simple pour les sédiments bruts non traités, déshydratés et traités avec les additifs.

Tableau 2. Résultats des essais de compression simple en fonction du temps

\begin{tabular}{cccc}
\hline Formule $/$ & Formulation F1 & Formulation F2 & Sédiment non traité \\
\cline { 2 - 4 } Temps (jours) & $R c(\mathrm{MPa})$ & $R c(\mathrm{MPa})$ & $R c(\mathrm{MPa})$ \\
\hline 7 & 2.03 & 3.21 & 0,64 \\
14 & 2.87 & 4.01 & 0,84 \\
28 & 2.80 & 4.06 & 0,83 \\
60 & 2.67 & 3.64 & 0,80 \\
\hline
\end{tabular}

La figure 4 montre que pour les 2 formulations étudiées, la résistance à la compression atteint des valeurs élevées dès 7 jours avec des valeurs supérieures aux exigences pour des utilisations en techniques de construction routière c'est-à-dire à 1 MPa.

En comparant les deux courbes des deux formulations sur la figure 4, nous pouvons conclure que l'augmentation de la résistance à la compression est essentiellement due à l'ajout du ciment. L'effet de la fumée de silice se manifeste à travers le taux d'augmentation de la résistance de la compression qui est de l'ordre de 3 par rapport aux échantillons de sédiments non traités par des liants (SILITONGA, 2010). Ceci peut être 


\section{XII ${ }^{\text {èmes }}$ Journées Nationales Génie Côtier - Génie Civil \\ Cherbourg, 12-14 juin 2012}

observé en comparant la courbe avec la formulation 3 et 5\% de ciment et les sédiments non traités.

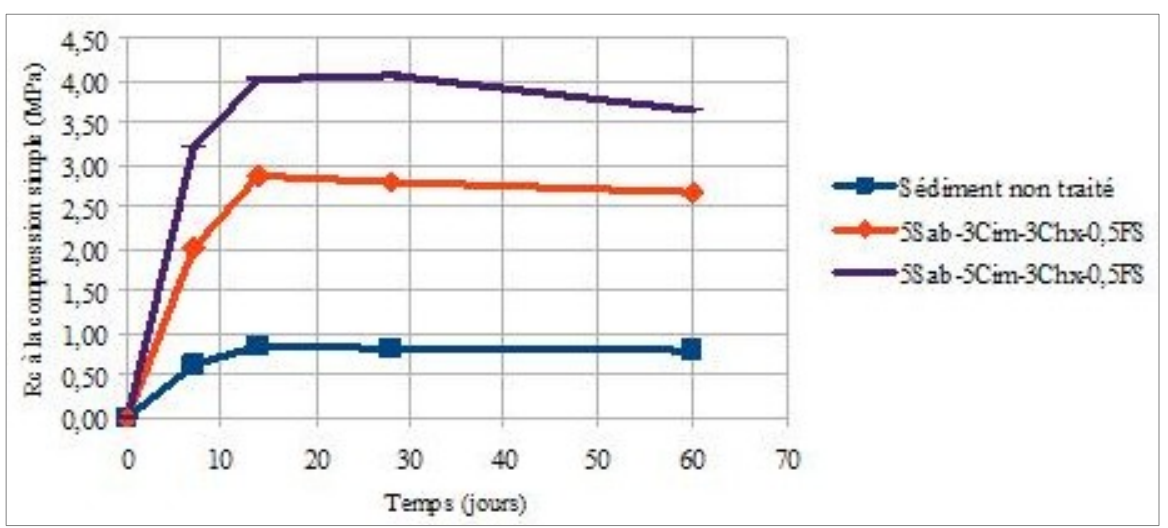

Figure 4. Résistance en compression simple des sédiments traités.

\subsection{Essai de durabilité}

La durabilité des sédiments traités est fortement influencée par la nature du sédiment, la porosité, le module d'élasticité, mais aussi par les conditions aux limites qui lui sont imposées et les liants utilisés (natures et dosages). Dans notre étude, la validation de la valorisation proposée pour les sédiments traités par solidification/stabilisation ne peut être envisagée que lorsque les conditions de comportement mécanique et environnemental à long terme sont assurées. La durabilité des sédiments solidifiés/stabilisés passe par la vérification de leur comportement à long terme.

Ces essais sont réalisés en laboratoire suivant des protocoles d'endommagement accélérés: la répétition des cycles d'endommagement sévères permet d'appréhender dans des délais courts (inférieurs à 2 mois) le comportement des matériaux sur plusieurs années. Ceci peut être évalué à l'aide des essais de comportement au gel/dégel, au mouillage/séchage et le relargage des métaux lourds à l’aide des essais de lixiviation.

\subsubsection{Essai de gel-dégel}

L'essai de gel/dégel est décrit par la norme NFP 98-234-1(1992) et consiste à comparer la résistance à la compression simple d'échantillons ayant subi 20 cycles de gel/dégel à celle des échantillons témoins de même âge.

Les réglementations normalisées exigent de tester les produits sous l'effet des cycles répétés de gel- dégel en laboratoire, en simulant des conditions d'humidité et de températures proches de celles que l'on rencontre généralement à la mise en œuvre, de telle sorte que les dégradations puissent apparaître lorsque la résistance au gel est insuffisante. Ce mode opératoire conduit à des résultats de laboratoire qui reproduisent bien le comportement in situ. 


\subsubsection{Effet du ciment sur la perte de masse}

Les résultats comparés des deux courbes de gel-dégel pour les deux formules montrent qu'une augmentation du ciment entraîne moins de perte de masse des échantillons, comme dans la formule F1 (5Sab-3Cim-3Chx-0.5FS), ce qui leur procure une meilleure résistance aux essais gel-dégel.

\subsubsection{Compression simple après essais gel-dégel}

L'essai permet de comparer la résistance en compression simple d'échantillons ayant subi des cycles de gel-dégel à celle des échantillons témoins du même âge. Nous avons effectué l'essai de gel-dégel sur 2 séries différentes. La première série permet une comparaison des résistances mécaniques en compression simple d'échantillons témoins âgés de 7 jours et ayant subi 21 cycles de gel-dégel avec des échantillons témoins du même âge et conservés à la température ambiante. La deuxième série permet une comparaison des résistances mécaniques en compression simple avec des échantillons témoins âgés de 28 jours et ayant subi 32 cycles de gel-dégel avec des échantillons témoins du même âge (60 jours) et conservés à la température ambiante. On présente ici uniquement les résultats de la première série. La figure 5 présente les pertes de résistance en compression simple dues aux cycles des gel-dégel.
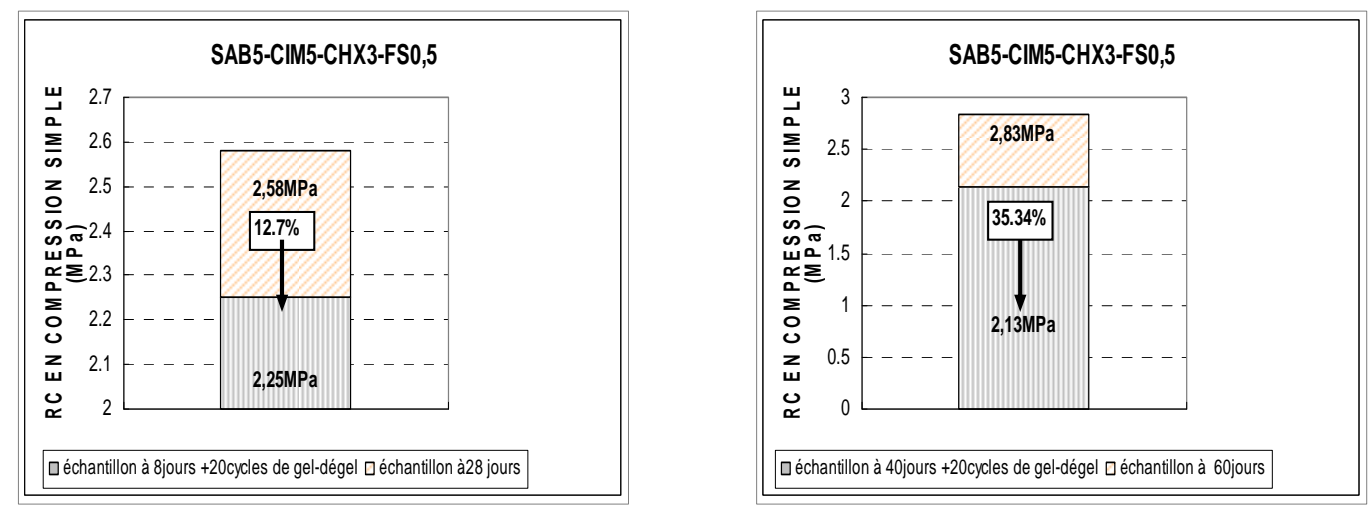

Figure 5. Comparaison de la résistance en compression après les essais de gel-dégel.

La comparaison entre les valeurs des résistances à la compression obtenues avec les 2 formulations pour les échantillons ayant subi des essais de gel/dégel et ceux de référence montre que les taux de diminution des valeurs de résistances pour les deux formulations sont plus importants pour les échantillons traités avec 3\% de ciment. Ceci signifie que la résistance les échantillons traités avec 5\% de ciment ont un meilleur comportement vis-à-vis du gel/dégel. Cependant, les résistances à la compression avec les deux formulations présentent des valeurs supérieures à $2 \mathrm{MPa}$. Ceci est largement supérieur aux valeurs exigées pour une utilisation en technique routière. Cela résulte essentiellement de l'effet de la fumée de silice et ce malgré son faible taux $(0,5 \%)$. 


\section{XII ${ }^{\text {èmes }}$ Journées Nationales Génie Côtier - Génie Civil \\ Cherbourg, 12-14 juin 2012}

Le bilan de ces résultats permet de conclure que la formulation à 3\% de ciment$3 \%$ chaux-0,5 fumée de silice-5\% sable est largement suffisante pour la valorisation du sédiment en couche de forme pour les techniques routières.

\subsubsection{Essais chimiques}

\subsubsection{Essais de lixiviation}

L'essai de lixiviation est défini par la norme française NF X-31-210 (1998), comme étant l'extraction liquide-solide, dans des conditions définies, d'un échantillon solide par une solution aqueuse.

La dégradation des échantillons stabilisés par les liants hydrauliques lors de la lixiviation est principalement liée à la diffusion des ions de l'eau interstitielle vers le milieu agressif, couplé à la dissolution progressive des hydrates. Cette diffusion ionique est donc le moteur de la dégradation des échantillons stabilisés.

Dans notre cas, on a choisi pour nos essais le test de lixiviation dynamique. L'eau déminéralisée a été utilisée avec un $\mathrm{pH}$ de 5 en tant que solution lixiviante. Dans une première phase, le dispositif a été rincé avec de l'acide nitrique dilué pour éviter toute contamination. L'échantillon cylindrique à 28 jours $(4 \times 8 \mathrm{~cm})$ repose sur un trépied en P.V.C. à $3,5 \mathrm{~cm}$ du fond. Les échantillons sont mis en contact avec de l'eau déminéralisée pendant 24 heures sous agitation permanente. Le $\mathrm{pH}$ du lixiviat est maintenu autour d'une valeur d'environ 5. A la fin de l'essai, le lixiviat est filtré puis analysé chimiquement.

\subsubsection{Analyse géochimique par l'essai de lixiviation dynamique}

Cet essai de lixiviation est une simulation du comportement du sédiment traité soumis à des attaques par des agents environnementaux extérieurs. Cet essai permet donc de valider les mélanges de valorisation des sédiments. Le choix de monolithe permet de se rapprocher le plus possible des conditions réelles des sédiments en chaussée routière. Ce type d'essai a permis d'identifier dans les sédiments étudiés sept métaux lourds constitués de zinc, sulfate, nickel, cadmium, chlorures, plomb, et cuivre. On présente ici quelques résultats.

Les résultats de l'analyse chimiques des lixiviats sont représentés graphiquement sur la figure 6 et comparés aux valeurs seuils du Conseil Européen n²003/33/CE (2003). Ce dernier a établi des valeurs de référence concernant les critères d'acceptation dans les décharges de déchets inertes, non-dangereux et dangereux. Ces valeurs concernent des éléments contenus dans les lixiviats et non dans le matériau brut. Si les valeurs analysées dans le lixiviat sont inférieures aux valeurs d'entrée en décharge pour déchets dangereux, on pourra considérer que les boues de dragage n'entrent pas dans la catégorie des déchets dangereux. 
Les sulfates : La figure 6 montre le taux de sulfates obtenu après l'essai de lixiviation. L'analyse montre que les mélanges ont un taux de sulfate de la catégorie des déchets inertes. L'excès de sulfate peut être dû au phénomène d'hydratation du ciment qui peut être modélisé par les réactions chimiques suivantes:

$$
\begin{array}{lll}
\mathrm{C}_{3} \mathrm{~A}+3 \mathrm{CSH}_{2}+26 \mathrm{H} & \Leftrightarrow & \mathrm{C}_{6} \mathrm{AS}_{3} \mathrm{H}_{32} \text { (ettringite) } \\
\mathrm{C}_{3} \mathrm{AS}_{3} \mathrm{H}_{32}+3 \mathrm{CA}+4 \mathrm{H} & \Leftrightarrow \quad 3 \mathrm{C}_{4} \mathrm{ASH}_{12} \text { (monsulfate) }
\end{array}
$$

Les deux forment des produits composés de sulfate de l'ettringite de formule $(\mathrm{CaO})_{6} \mathrm{Al}_{2} \mathrm{O}_{3}\left(\mathrm{SO}_{3}\right)_{3}\left(\mathrm{H}_{2} \mathrm{O}\right)_{32}$ pour l'équation (1) et pour l'équation (2), monosulfate de formule $(\mathrm{CaO})_{4} \mathrm{Al}_{2} \mathrm{O}_{3} \mathrm{SO}_{3}\left(\mathrm{H}_{2} \mathrm{O}\right)_{12}$ (SILITONGA, 2010). Mais ce taux excessif de sulfate diminue avec l'ajout de $1 \%$ de fumée de silice. On peut donc conclure que l'ajout de fumée de silice a une action positive sur la diminution du taux de sulfate.

Le zinc: La figure 6 donne la valeur de zinc mesurée pour les deux mélanges. On constate que la valeur de zinc se situe dans la zone des déchets inertes. L'effet du traitement avec différente composition de liants provoque une diminution de la valeur de zinc par apport au sédiment non traité. Sur la figure 6 on observe que le mélange (formulation F1) possède une valeur de zinc supérieure à celle de la formule (formulation F2). L'ajout de fumée de silice diminue la valeur de zinc en fixant les métaux lourds grâce à ses fines particules (SILITONGA et al., 2010 ; BEHMANESH 2008). On confirme que l'ajout de fumée de silice a un effet positif car il a entraîné une réduction du taux de zinc pour tous les mélanges étudiés.

Le nickel : La figure 6 montre les taux de nickel pour les deux mélanges et permet de les classer dans les catégories des déchets inertes. L'analyse des deux traitements montre que ces taux sont légèrement supérieurs au sédiment non traité. Cette légère différence est due à la diversité de la composition du sédiment utilisé. En effet, comme expliqué précédemment, le sédiment a subi une pré- étape de séchage qui a fait varier leur teneur en eau initiale. Par conséquent, les caractéristiques des sédiments et le taux des métaux lourds a dû être modifiées.

Le plomb : L'effet des liants sur la réduction du taux de plomb est clairement effectif. La figure 6 montre que les taux de plomb de tous les mélanges traités ont été réduits par rapport au sédiment sans traitement. L'effet de la fumée de silice et du ciment sur la réduction du relargage des métaux lourds, notamment pour le plomb, est largement démontré.

\section{Conclusions}

L'étude réalisée a permis de proposer une solution de valorisation des sédiments de dragage du port d'Honfleur pour une possible utilisation dans les techniques de construction routière. La fumée de silice améliore la structure physique des sédiments traités en remplissant les pores, et augmente ainsi la densité des échantillons. La présence de la fumée de silice conduit donc à une réduction de la porosité ce qui augmente la résistance de la matière cimentaire. 


\section{XII ${ }^{\text {èmes }}$ Journées Nationales Génie Côtier - Génie Civil \\ Cherbourg, 12-14 juin 2012}
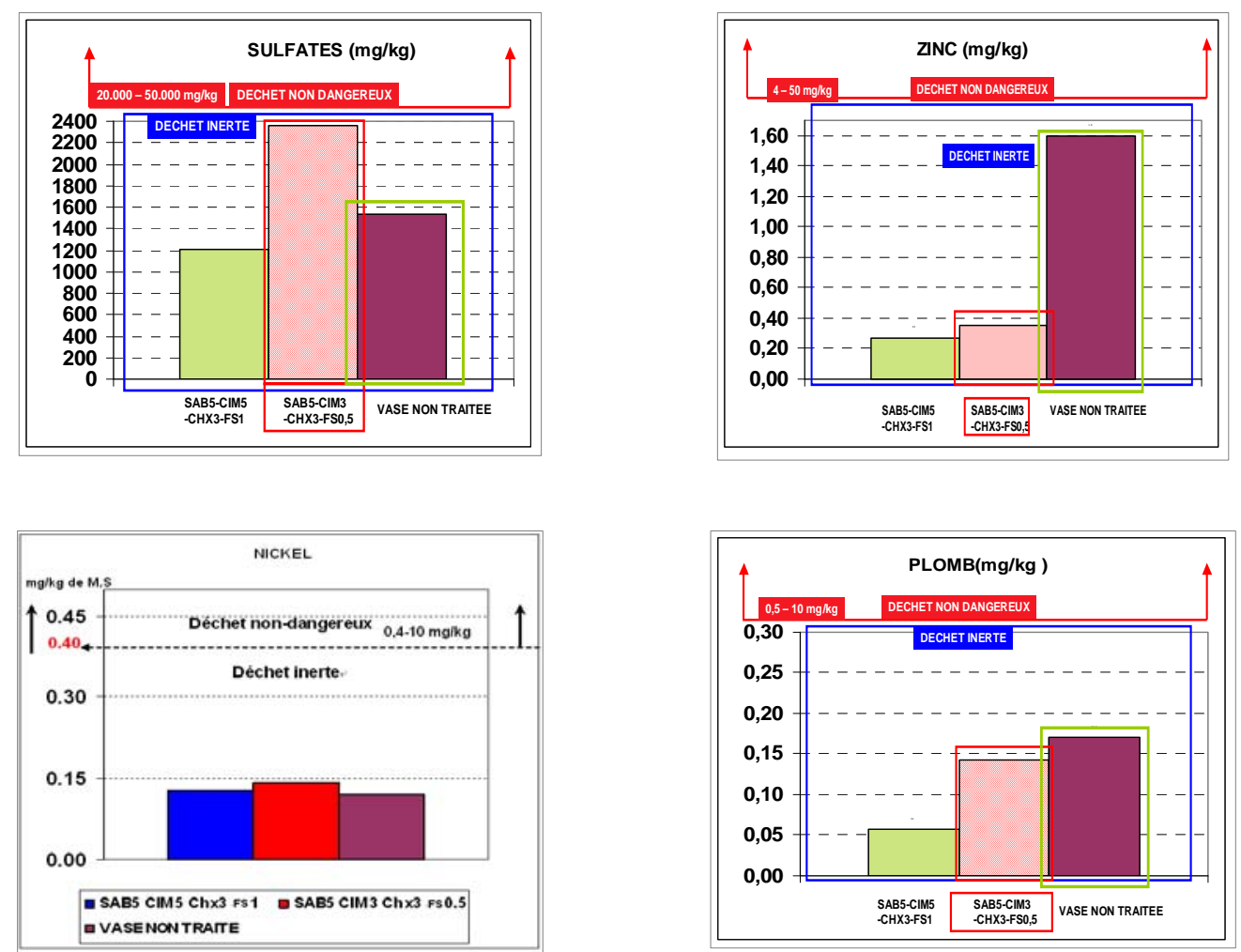

Figure 6. Concentration des polluants obtenus après l'essai de lixiviation dynamique.

L'ajout de la chaux va également accroître les caractéristiques mécaniques, mais sur une période plus longue en comparaison du ciment grâce aux réactions pouzzolaniques. Il semble que la chaux ait un effet bénéfique malgré le faible dosage utilisé dans notre étude (3\%). L'autre paramètre important, caractéristique de la durabilité d'un matériau, est la résistance au gel. Dans le cas de notre étude, l'essai réalisé est l'essai de résistance au gel-dégel (NF P 98-234-1). La présence de fumée de silice et du ciment dans les formulations a permis d'obtenir des résultats intéressants vis-à-vis des critères de durabilité et de conditions défavorables d'exposition des matériaux.

Des essais de lixiviation ont été également réalisés selon un programme expérimental sur monolithes d'après la norme NF X31-210 (1998). Les essais ont été réalisés à 60 jours, sur des échantillons de chaque formulation. Les résultats sont comparés à législation relative à la mise en centre de stockage des déchets (Décision européenne 2003/33/CE du 19/02/2002). Les résultats montrent que pour les formulations étudiées sur la base des paramètres physico-chimiques, les matériaux sont considérés non dangereux à inertes. La comparaison des résultats avec les sédiments après traitement, pour les deux formules étudiées, permet d'affirmer que l'augmentation du taux de ciment et de fumée silice, engendre une réduction du taux de relargage des métaux lourds. 


\section{Références bibliographiques}

AFNOR NF P 94-068 (1998). Sols: reconnaissance et essais, «Mesure de la capacité d'adsorption de bleu de méthylène d'un sol ou d'un matériau rocheux - Détermination de la valeur de bleu de méthylène d'un sol ou d'un matériau rocheux par l'essai à la tache. $7 \mathrm{p}$.

AFNOR NF P 98-234-1 (1992). Comportement au gel des matériaux traités aux liants hydrauliques, Partie 1: Essai de résistance au gel-dégel des graves et sables traités, 6 p. AFNOR NF X 31-210 (1998). Déchets «Essai de lixiviation». 16 p.

BAI X. (2010). Caractérisation physico-chimique des sédiments de dragage du port en Bessin. Mémoire de Master 2 Recherche, Sciences de l'environnement, Université de Caen, 62 p.

BEHMANESH J. (2008). Etude de la durabilité d'un sédiment, traité au ciment et additifs. Thèse de doctorat, Université de Caen, $216 \mathrm{p}$.

Décision européenne 2003/33/CE (2002). Etablissant des critères et des procédures d'admission des déchets dans les décharges, conformément à la directive décharge 1999/31/CE.

GTR (1992).Guide technique pour la réalisation des remblais et des couches de forme. Fascicule I, principes généraux, 100 p.

LEI X. (2011). Etude de la durabilité des sédiments du port d'Honfleur traité aux ciments et additifs. Mémoire de Master 2 Recherche, Master Sciences de l'environnement, $63 \mathrm{p}$.

SILITONGA E., MEZAZIGH S., LEVACHER D. (2010). Etude de la durabilité d'une valorisation de sédiments marins traités avec différents types de fumée de silice. Revue Européenne de Génie Civil et Environnement, Vol. 14, n 2, pp 253-265. doi:10.3166/ejece.14.253-265

SILITONGA E. (2010). Valorisation des sédiments marins contaminés par solidification/stabilisation à base de liants hydrauliques et fumée de silice. Thèse de doctorat, Université de Caen, 216 p. 